\title{
Use Piecewise Crow-AMSAA Method to Predict Infection and Death of Coronavirus in Iran
}

\author{
P. Gholami ${ }^{*}$, S. Elahian ${ }^{2}$ \\ 1. Department of Aerospace Engineering, Sharif University of Technology, Tehran, Iran \\ 2. Aerospace Research Institute(Ministry of Science, Research and Technology), Tehran, Iran
}

\begin{abstract}
Reliability growth is the positive improvement in a product's criteria (or parameter) over a period of time due to changes in the design or product process. By analyzing the growth of reliability in a system, it can be seen that at a certain stage of the epidemic, the growth of the transmission and the rate of infection change over time. During the spread of disease, problem areas are identified and knowledge of the disease increased and then initial treatment and tools may be redesigned or reprocessed to take appropriate corrective action. In other words, each stage of the spread of the disease has a different level of growth transmission depending on appropriate corrective action. Therefore, according to this case, there are conditions under which phenomena can be described by Non-Homogeneous Poisson Process (NHPP). However, traditional epidemiological models based on exponential distribution are not able to predict disease growth during different stages of the outbreak. Therefore, in this paper, the Piecewise Crow-AMSAA (NHPP) model, which is based on the Non-Homogeneous Poisson process, is used to predict the growth of infected cases and deaths of Coronavirus outbreak. Initially, the Iran cumulative confirmed case and death data are divided into several sections based on the manual separation to find out each different infection phase at each different time period. Then Crow-AMSAA (NHPP) model is applied to the segmented data. At each stage of the outbreak, the model parameters are estimated independently using the maximum likelihood estimation (MLE) technique. Finally, the growth parameters in each stage are compared with each other and external and environmental factors are identified and examined.
\end{abstract}

Keyword: Coronavirus, Non-Homogeneous Poisson Process (NHPP), Piecewise Crow-AMSAA (NHPP), Infected cases, Deaths.

\section{Nomenclature}

E $\quad$ Expected number

$n \quad$ Number of confirmed infected cases or deaths

Cumulative confirmed infected cases or deaths

$N$

$\begin{array}{ll}t & \text { Cumulative test time } \\ T & \text { Test interval time }\end{array}$

$T$

$\beta$

Growth parameter of Crow-AMSAA

(NHPP)

(speed of the transmission or death rate)

$\rho \quad$ Confirmed infected cases or deaths occurring rate

Scale parameter of Crow-AMSAA (NHPP)

$\lambda$ (instantaneous confirmed infected cases or deaths intensity)

Cumulative confirmed infected cases or

$\lambda_{c} \quad$ deaths rate

NHPP Non-Homogeneous Poisson Process

MLE Maximum likelihood estimation

pdf probability density function

\footnotetext{
* Corresponding Author Email: p.gholami@ae.sharif.ir
}

\section{Introduction}

To improve system functionality, the first step is to analyze the system and detect its defects. Then it can be decided how to eliminate them and improve the system or grow its reliability. The growth can be modeled in different methods to predict the new behavior of the system after promotion. In this article, the pandemic spread is considered as a system and any new cognition about the virus such as its properties and features, that helps specialists to improve methods for dealing with the disease or each new policy that helps to change the trend of the outbreak, cause an improvement in system functionality and its prediction model. In the article, the model used in each phase of disease spread is developed by calculation of its parameters according to modeling methods.

The novel Coronavirus named Coronavirus Disease 2019 or COVID-19 is pandemic and as reported by "WHO", for the first time it had seen in Wuhan, China in December 2019. High rate of COVID-19 spreading and unknown parameters affected on it, increase the worthiness of spreading model which helps governments 
to behave more accurate and decide timely decision to deal with disease and cease outbreak. So, different papers have focused on COVID-19 to predict rate of disease spread and modeling it by different methods.

Car, et al. [1] used dataset of 51 days for training multi-layer perception artificial neural network to develop a model for maximum number of infections prediction. Prediction model has a good robustness for confirmed cases and is low for recovered ones.

$\mathrm{Li}$, et al. [2] survey the process of COVID-19 transmission in China. They use Gaussian distribution for transmission stage modeling. According to the article, simulation results show good estimation model in comparison with the official data.

In the other work, COVID-19 spreading is surveyed if initial stage of disease outbreak. Catala, et al. [3] apply Gompertz function to model new infection cases. According to their results, suggested model has good results for short-time prediction. They noted that, spreading velocity in initial stage of epidemiological disease varies systematically for different countries.

In the other work Zareie, et al. [4] applied Susceptible-infectious-recovered (SIR) model for COVID-19 estimation in Iran based on China parameters. Using estimation of three time-dependent parameters including transmission, death, and recovery rate from China dataset besides number of infections in Iran, they predicted number of patients in Iran for the next month. Generalized Additive model (GAM) is used for the estimation of the parameters.

Reliability growth models are models used to estimate or predict the improvement of the reliability of a complex system as a function of the number of experimental tests. Reliability growth modeling began with empirical observations by Duane [5] on developmental testing programs for relatively complex aircraft accessories. For the systems he was tracking, on a $\log -\log$ scale, the cumulative number of failures tended to increase linearly with the cumulative test time. Since then, many reliability growth models have been developed. Crow [6-8] noted that the Duane model could be stochastically represented as a Weibull process, allowing for statistical procedures to be used in the application of this model in reliability growth. This statistical extension became what is known as the CrowAMSAA (NHPP) model. This method was first developed at the U.S. Army Materiel Systems Analysis Activity (AMSAA). In recent years, there has been much effort to develop the Crow-AMSAA (NHPP) model and create better models for mechanical systems [9-13]. Wang et al. [14]studied a reliability growth testing allocation problem to series-parallel systems that consider parameter uncertainty in the Crow-AMSAA (NHPP) model. They assumed the parameters of the model to be known as uncertain-but-bounded values.Lee and his colleagues [15] used the Crow-AMSAA (NHPP) model to analyze the reliability growth of multiple launch rocket components by test data obtained from the development phase. Recently, the CrowAMSAA(NHPP) model was developed by Nadjafi and Gholami[16]using the normal distribution and maximum likelihood estimate (MLE) and proved its effectiveness on the data of an aerospace system.

The development of an event may consist of several separate stages. The Crow-AMSAA (NHPP) model has been modified to track reliability at each stage of the event. Hence, Piecewise Crow-AMSAA (NHPP) is introduced to fit an independent growth model to data from each stage of development.In this work, the CrowAMSAA (NHPP) and Piecewise Crow-AMSAA (NHPP)reliability growth models are used to predict the growth of infection and the death of Coronavirus.Unknown growth parameter $\beta$ of the CrowAMSAA (NHPP) model is used as the growth parameter of infected cases or deaths. The traditional epidemiological model is based on the exponential distribution, but the Crow-AMSAA is the NonHomogeneous Poisson Process (NHPP) which can be used to model the complex problem like Coronavirus, especially when the various mitigation strategies and correction actions were implemented by the government at different phases. Ranjan [17], Canabarro, et al. [18] and Liu, et al. [19] are all using the traditional epidemiological model to predict the spreading the Coronavirus. The traditional epidemiological models are very difficult to predict the numbers of infections when the disease spreading enters to a new different phase, in addition these methods have too many parameters and assumptions, and it is susceptible to the parameters and assumptions.

The Crow-AMSAA (NHPP) and Piecewise CrowAMSAA (NHPP) model were trialed to make predictions of Iran infected cases and deaths from $2 / 19 / 2020$ to $9 / 10 / 2020$ is used. At first, it is assumed that the cumulative data is not grouped and the CrowAMSAA (NHPP) model is applied to it. In the next step, using the cumulative and daily curve, the cumulative curve is divided into several sections and then the Piecewise Crow-AMSAA (NHPP) model is implemented on grouped data. It is worth noting that the maximum likelihood estimation (MLE) algorithm is used to estimate the growth parameter of the model $(\beta)$ with experimental data on infected and death due to Coronavirus. In this method, unknown parameters are estimated in such a way that the likelihood function (LF) of the observed data is maximized. After estimating the parameters, cumulative curves of infected and death events are plotted and compared with the experimental data obtained from Coronavirus tests, and the efficiency of the model in predicting these events is shown. Finally, the results obtained from the data of Iran are compared with the data of China and the growth of infected cases or deaths of these two countries are examined. 


\section{Crow-AMSAA(NHPP)}

The reliability growth pattern for the Crow-AMSAA (NHPP) model is exactly the same pattern as for the Duane postulate, that is, the cumulative number of failures is linear when plotted on $\ln -\ln$ scale. If $N(t)$ be the cumulative confirmed infected cases or deaths in cumulative test time $t$, and $\rho(t)$ be the confirmed infected cases or deaths occurring rate for the Crow-AMSAA (NHPP) model, then the expected number of confirmed infected cases or deaths experienced over the test interval $[0, T]$ under the Crow-AMSAA (NHPP) model is given by:

$$
E[N(T)]=\int_{0}^{T} \rho(t) d t
$$

The Crow-AMSAA (NHPP) model assumes that $\rho(T)$ may be approximated by the Weibull failure rate function:

$$
\rho(T)=\frac{\beta}{\eta^{\beta}} T^{\beta-1}
$$

Therefore, if the intensity function $\rho(T)$, or the instantaneous confirmed infected cases or deaths intensity, $\lambda_{i}(T)$, is defined as:

$$
\lambda_{i}(T)=\lambda \beta T^{\beta-1}, \quad T>0, \lambda>0, \beta>0
$$

where $\lambda$ is the scale parameter and $\beta$ is the growth parameter. If the parameter $\beta$ is greater than 1 , the transmission rate is increasing, the transmission rate is more rapid, if the parameter $\beta$ is smaller than 1 , the transmission rate is decreasing, the transmission rate is slower. In the special case of exponential confirmed infected cases or deaths times, there is no growth and the failure intensity, $\rho(t)$, is equal to $\lambda$. In this case, the expected number of confirmed infected cases or deaths is given by:

$$
\begin{aligned}
E[N(T)] & =\int_{0}^{T} \rho(t) d t \\
& =\lambda T
\end{aligned}
$$

In order for the plot to be linear when plotted on lnIn scale under the general reliability growth case, the following must hold true where the expected number of confirmed infected cases or deaths is equal to:

$$
\begin{aligned}
E[N(T)] & =\int_{0}^{T} \rho(t) d t \\
& =\lambda T^{\beta}
\end{aligned}
$$

To put a statistical structure on the reliability growth process, consider again the special case of no growth. In this case the number of failures $N(T)$, experienced during the testing over $[0, T]$ is random. The expected number of confirmed infected cases or deaths $N(T)$, is said to follow the homogeneous (constant) Poisson process with mean $\lambda T$ and is given by:

$$
\operatorname{pr}[N(T)=n]=\frac{(\lambda T)^{n} e^{-\lambda T}}{n !} ; n=0,1,2, \ldots
$$

The Crow-AMSAA (NHPP) model generalizes this no growth case to allow for reliability growth due to corrective actions. This generalization keeps the Poisson distribution for the number of failures but allows for the expected number of confirmed infected cases or deaths
$E[N(T)]$, to be linear when plotted on $\ln -\ln$ scale. The Crow-AMSAA (NHPP) model lets $E[N(T)]=\lambda T^{\beta}$. The probability that the number of confirmed infected cases or deaths, $N(T)$ will be equal to $n$ under growth is then given by the Poisson distribution:

$\operatorname{pr}[N(T)=n]=\frac{\left(\lambda T^{\beta}\right)^{n} e^{-\lambda T^{\beta}}}{n !} ; n=0,1,2, \ldots$

This is the general growth situation, and the number of confirmed infected cases or deaths, $N(T)$, follows a Non-Homogeneous Poisson process. The exponential, "no growth" Homogeneous Poisson Process is a special case of the Non-Homogeneous CrowAMSAA (NHPP) model. This is reflected in the CrowAMSAA (NHPP) model parameter where $\beta=1$. The cumulative confirmed infected cases or deaths rate, $\lambda_{c}$, is:

$\lambda_{c}=\lambda T^{\beta-1}$

\section{Piecewise Crow-AMSAA (NHPP)}

In this method Crow-AMSAA (NHPP) models are fit to each of phases under the constraint that the cumulative number of events at the start of a phase matches withnumber at the end of the preceding phase. Therefore, with this method, the transmission growth parameter is calculated at each stage. This model allows us to compare each stage of the event independently.Therefore, at each stage of the event, the model parameters are calculated independently and the growth rate and deterioration of transmission are compared with each other and the external and environmental factors involved in this growth and deterioration are identified and examined.

\section{Parameter Estimation}

In reliability analysis, the parameters of the reliability growth model based on the Non-Homogeneous Poisson process should be estimated from the observed data.The usual method for parameter evaluation is to estimate maximum likelihood estimation (MLE).There are multiple data entry schemes for this data type and each is presented next.

Grouped data type is used for tests where the exact confirmed infected cases or death times are unknown and only the number of confirmed infected cases or deaths within a time interval is recorded. But Nongrouped data is used when confirmed infected cases or death times are known and obvious. So, in the CrowAMSAA model, Non-grouped data was used, while in the Piecewise Crow-AMSAA model, grouped data was used.

\section{Non-Grouped data}

The probability density function (PDF) of the $i^{\text {th }}$ event given that the $(i-1)^{\text {th }}$ event occurred at $T_{i-1}$ is: 
$f\left(T_{i} \mid T_{i-1}\right)=\frac{\beta}{\eta}\left(\frac{T_{i}}{\eta}\right)^{\beta-1} \cdot e^{-\frac{1}{\eta^{\beta}}\left(T_{i}^{\beta}-T_{i-1}^{\beta}\right)}$

Assuming that $\lambda=1 / \eta^{\beta}$, the likelihood function is:

$L=\lambda^{n} \beta^{n} e^{-\lambda T^{* \beta}} \prod_{i=1}^{n} T_{i}^{\beta-1}$

Where $T$ is the termination time and taking the natural $\log$ on both sides:

$\Lambda=n \ln \lambda+n \ln \beta-\lambda T^{\beta}+(\beta-1) \sum_{i=1}^{n} \ln T_{i}$

Differentiating with respect to $\lambda$ and $\beta$, and set equal to zero yields:

$$
\begin{aligned}
& \frac{\partial \Lambda}{\partial \lambda}=\frac{n}{\lambda}-T^{\beta} ; \quad \hat{\lambda}=\frac{n}{T^{\beta}} \\
& \frac{\partial \Lambda}{\partial \beta}=\frac{n}{\beta}-\lambda T^{\beta} \ln T^{\beta}+\sum_{i=1}^{n} \ln T_{i} ; \quad \hat{\beta}=\frac{n}{n \ln T-\sum_{i=1}^{n} \ln T_{i}}
\end{aligned}
$$

\section{Grouped data}

This type of data is used for tests where the exact confirmed infected cases or deaths times are unknown and only the number of confirmed infected cases or deaths within a time interval are recorded (e.g., daily Coronavirus tests).

For analyzing grouped data, if the equation $E[N(T)]$ from the Equation (5) is linearized:

$\ln E[N(T)]=\ln \lambda+\beta \ln T$

According to Crow-AMSAA (NHPP), the likelihood function for the grouped data case is:

$$
\prod_{i=1}^{k} \operatorname{pr}\left(N_{i}=n_{i}\right)=\prod_{i=1}^{k} \frac{\left(\lambda T_{i}^{\beta}-\lambda T_{i-1}^{\beta}\right)^{n_{i}} \cdot e^{-\left(\lambda T_{i}^{\beta}-\lambda T_{i-1}^{\beta}\right)}}{n_{k} !}
$$

Where $n_{1}, n_{2}, \ldots, n_{k}$ are confirmed infected cases or deaths and $k$ is the number of days. The MLE of $\lambda$ based on this relationship is:
$\hat{\lambda}=\frac{n}{T^{\dot{\beta}}}$

Where $n$ is the total number of confirmed infected cases or deaths from all the days. The estimate of $\beta$ satisfies:

$\sum_{i=1}^{k} n_{i}\left[\frac{T_{i}^{\hat{\beta}} \ln T_{i}-T_{i-1}^{\hat{\beta}} \ln T_{i-1}}{T_{i}^{\hat{\beta}}-T_{i-1}^{\hat{\beta}}}-\ln T_{k}\right]=0$

\section{Result and discussion}

Dataset to develop the model, get from World Health Organization (WHO) Coronavirus data. It contains data of new confirmed cases, new death, cumulative confirmed cases, and cumulative death for Iran and China (the source of disease) over 210 days.

The classical approach to reliability growth estimation normally is in the form of a mathematical formula with one or more parameters to be estimated from the data collected at one stage of development.

In this method, Crow-AMSAA (NHPP) models are fit to each of the phases under the constraint that the cumulative number of events at the start of a phase matches that number at the end of the preceding phase. Therefore, with this method, the transmission growth parameter is calculated at each stage. This model allows us to compare each stage of the event independently.

Crow-AMSAA (NHPP) and Piecewise CrowAMSAA (NHPP) models based on the Ln-Ln equations are applied to the experimental data obtained from Coronavirus tests, which are shown in Figs. 1 \& 2 for theconfirmed infected cases or deaths, respectively.

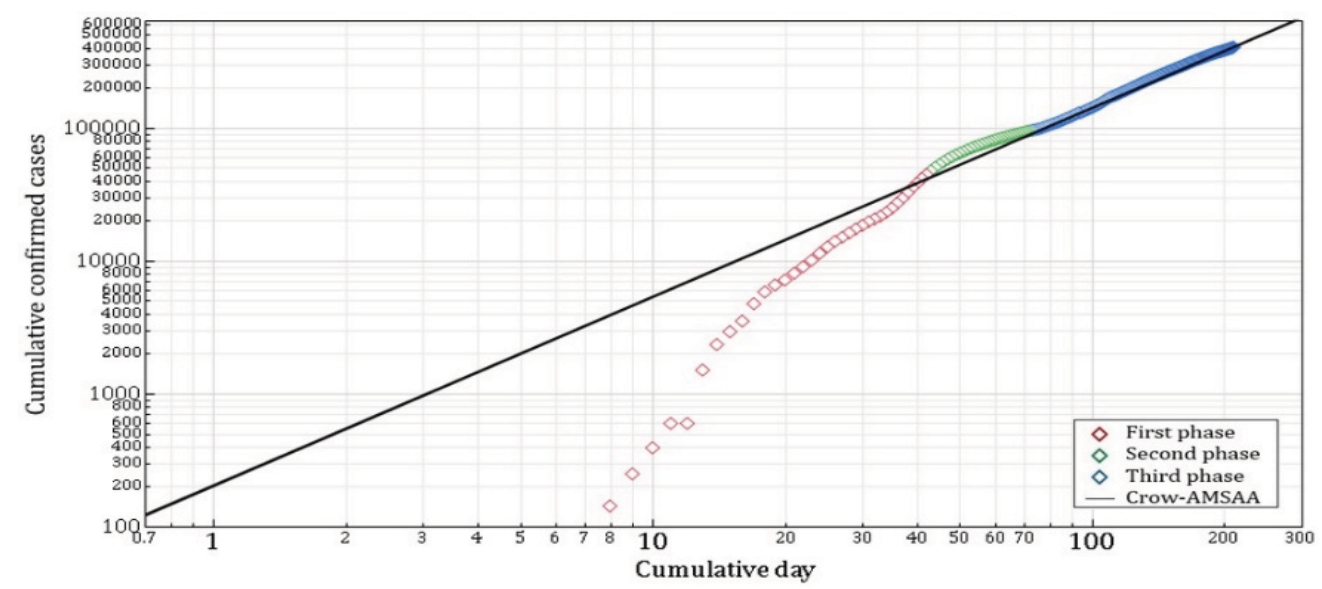

(a) 


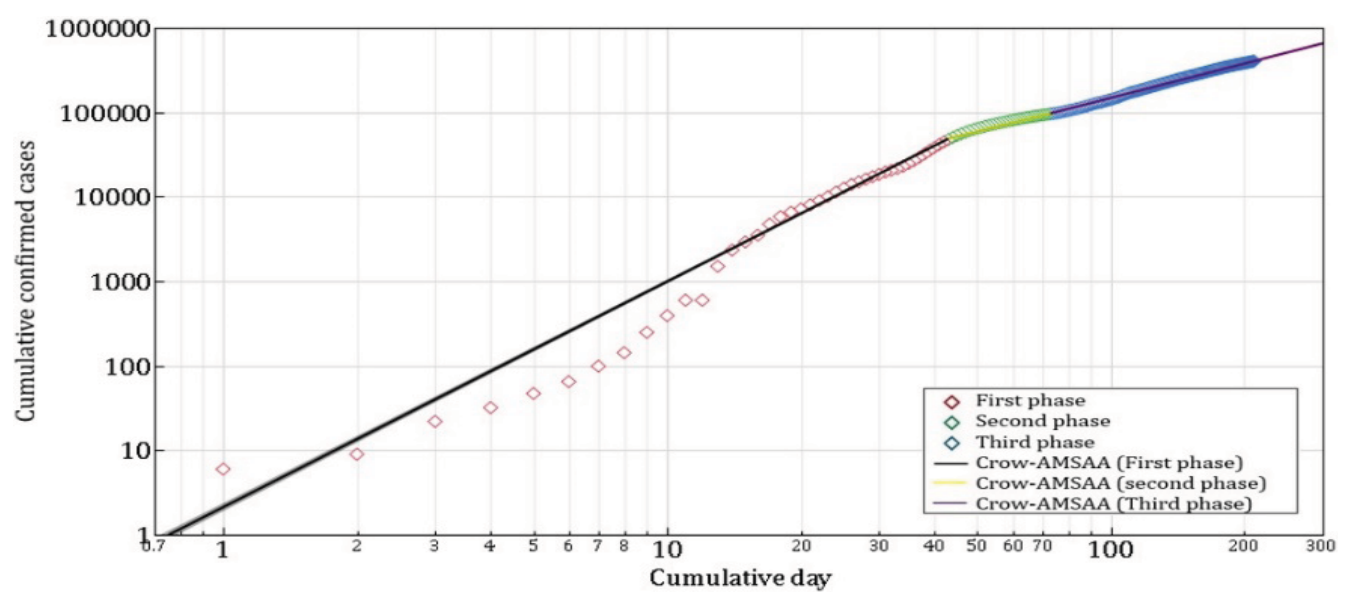

(b)

Figure 1. Cumulative confirmed infected case of Iran; (a) Crow-AMSAA model, (b) Piecewise Crow-AMSAA model

Then, according to the Non-Grouped and Grouped data, by using the maximum likelihood estimation (MLE) algorithm, the values of $\lambda$ and $\beta$ for each model are obtained and shown in Table $1 \& 2$. In the CrowAMSAA (NHPP) model, it is assumed that the number of daily confirmed infected cases or deaths are neglected, and each of the recorded days is considered as days of confirmed infected cases or deaths. In other words, the data observed in this model are assumed to be Non-Grouped and the values of $\lambda$ and $\beta$ are obtained using these data. But in the Piecewise Crow-AMSAA (NHPP) model, the test time is considered 24 hours, and the number of confirmed infected cases and deaths as can be seen from the experimental data, varies over the test period. In other words, it is considered as Grouped data and with this assumption and according to number of phases of observed cumulative data, the values of $\lambda$ and $\beta$ in each phase are obtained.

On the other hand, to show the growth speed of the transmission and death rates, the parameters obtained from daily reported data from Iran are compared with the estimated parameters of the data from Chinese infected cases and deaths as the country of origin of the Coronavirus outbreak. The daily confirmed case and death rate for Coronavirus in China is plotted in Figs. 3 $\& 4$, respectively, and the values of $\lambda$ and $\beta$ for each model are obtained and shown in Table $1 \&$ 2.It should be noted that the Fisher matrix approach is used to estimate the confidence bonds of the parameters of the Crow-AMSAA (NHPP) and Piecewise Crow-AMSAA (NHPP) models.

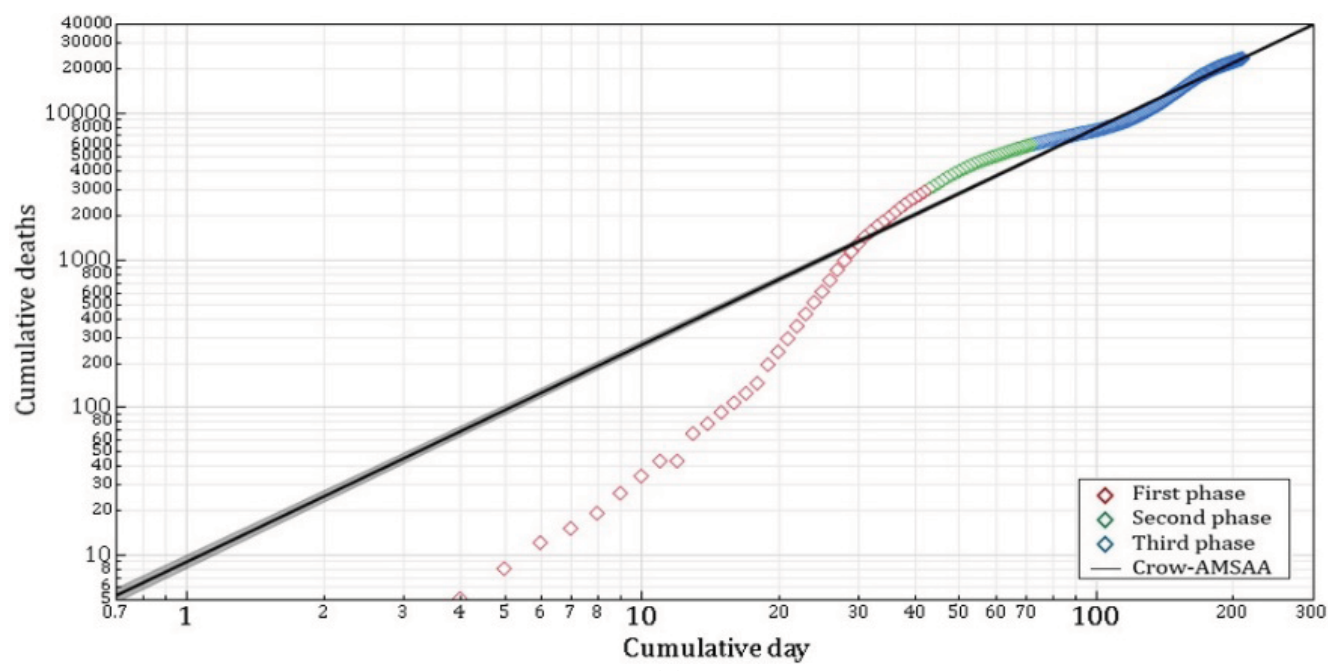

(a) 


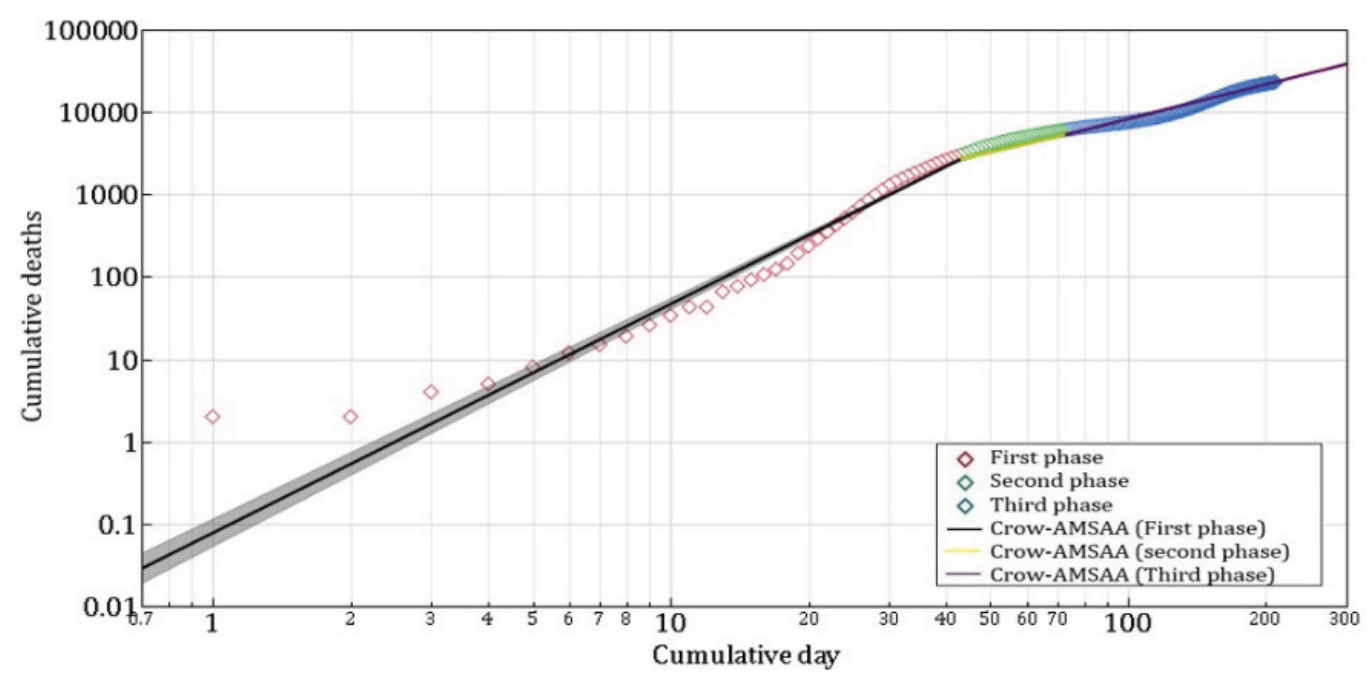

(b)

Figure 2. Cumulative death of Iran; (a) Crow-AMSAA model, (b) Piecewise Crow-AMSAA model

Table 1.Estimation of Crow-AMSAA and Piecewise Crow-AMSAA model parameters based on the confirmed infected case data

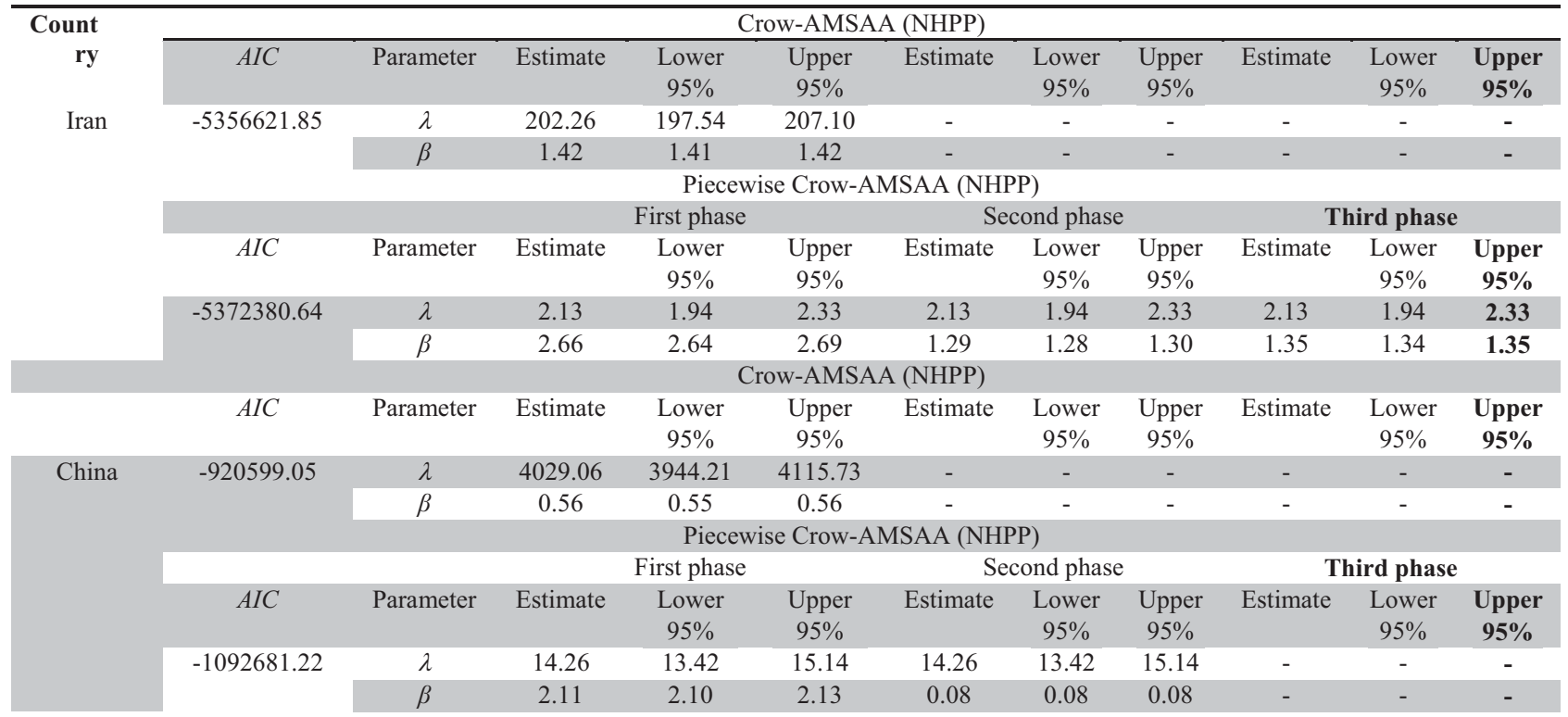

Table 2.Estimation of Crow-AMSAA and Piecewise Crow-AMSAA model parameters based on the death data

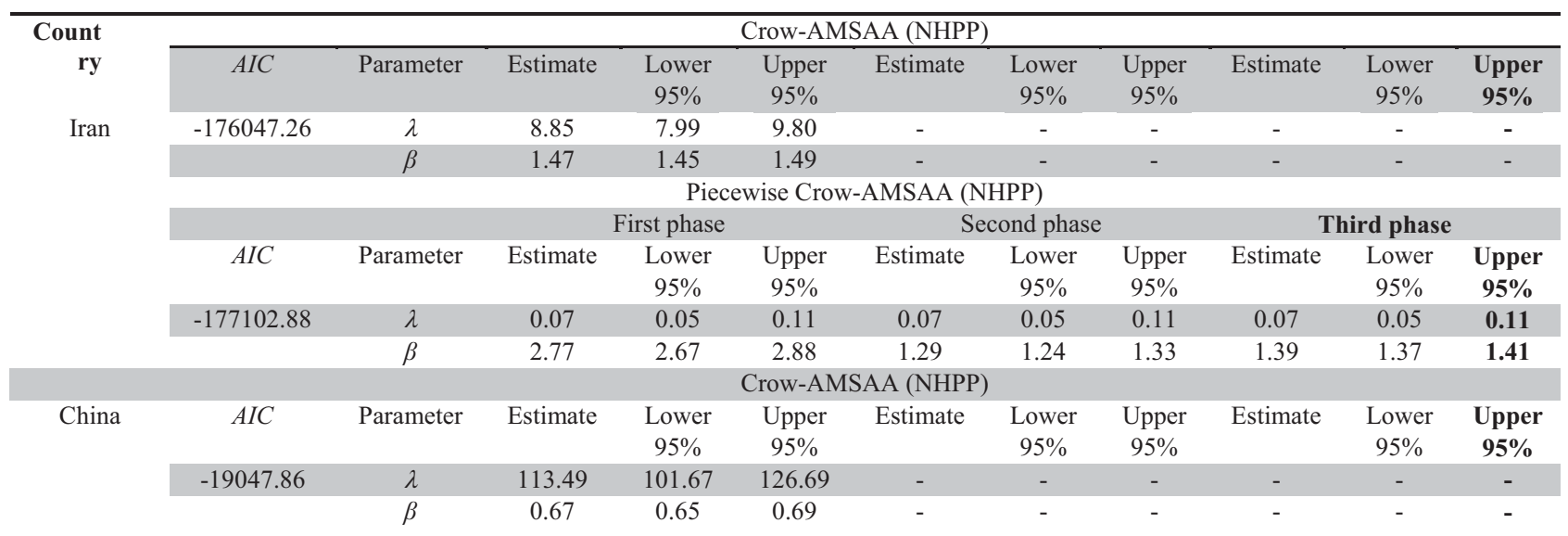




\begin{tabular}{|c|c|c|c|c|c|c|c|c|c|c|}
\hline \multicolumn{11}{|c|}{ Piecewise Crow-AMSAA (NHPP) } \\
\hline \multirow[b]{2}{*}{$A I C$} & \multirow[b]{2}{*}{ Parameter } & \multicolumn{3}{|c|}{ First phase } & \multicolumn{3}{|c|}{ Second phase } & \multicolumn{3}{|c|}{ Third phase } \\
\hline & & Estimate & $\begin{array}{c}\text { Lower } \\
95 \%\end{array}$ & $\begin{array}{c}\text { Upper } \\
95 \%\end{array}$ & Estimate & $\begin{array}{c}\text { Lower } \\
95 \%\end{array}$ & $\begin{array}{c}\text { Upper } \\
95 \%\end{array}$ & Estimate & $\begin{array}{c}\text { Lower } \\
95 \%\end{array}$ & $\begin{array}{c}\text { Upper } \\
95 \%\end{array}$ \\
\hline-24307.33 & $\lambda$ & 0.03 & 0.02 & 0.05 & 0.03 & 0.02 & 0.05 & - & - & - \\
\hline & $\beta$ & 2.77 & 2.67 & 2.88 & 0.31 & 0.29 & 0.32 & - & - & - \\
\hline
\end{tabular}

To choose the best model among the abovementioned, in this study, the Akaike Information Criterion $(A I C)$ is used. The $A I C$ is defined by $A I C=-2 L(S)+2 P$, where $L$ is the maximum of the likelihood function of the model, while $S$ and $P$ are the number of observations and number of parameters, respectively. Lower $A I C$ values are better, and $A I C$ penalizes models that use more parameters. So, if two models explain the same amount of variation, the one with fewer parameters will have a lower $A I C$ value and will be the better-fit model.

For this purpose, for the data of infected cases and deaths, this criterion is estimated and compared with different modelsaslisted in Tables 1 \& 2.As shown in Tables $1 \& 2$, the Piecewise Crow-AMSAA (NHPP) model has the smallest $A I C$ value, which is an indicator of a good fit. So, the $\beta$ values of this model are used to study the speed of the transmission or death rate due to this outbreak for each phase and to determine the related factors. As mentioned earlier, if $\beta$ parameter is greater than 1 , the speed of transmission or death rate increases, and if the growth parameter $\beta$ is less than 1 , the speed of transmission or death rate decreases.

As can be seen from the data related to Iran and Table 1, the growth parameter of $\beta$ in all phases is greater than 1, which means that the speed of the transmission in all stages is increasing. But in the second phase of the outbreak in Iran, the value of the growth parameter $\beta$ is equal to 1.29 , which is smaller than the

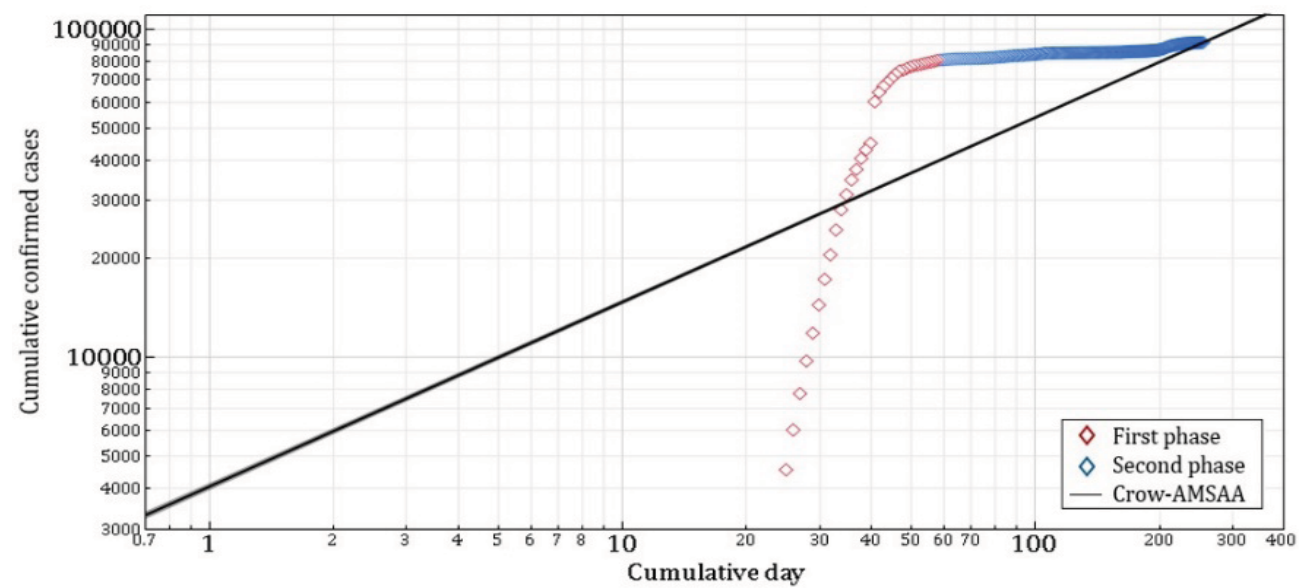

(a)

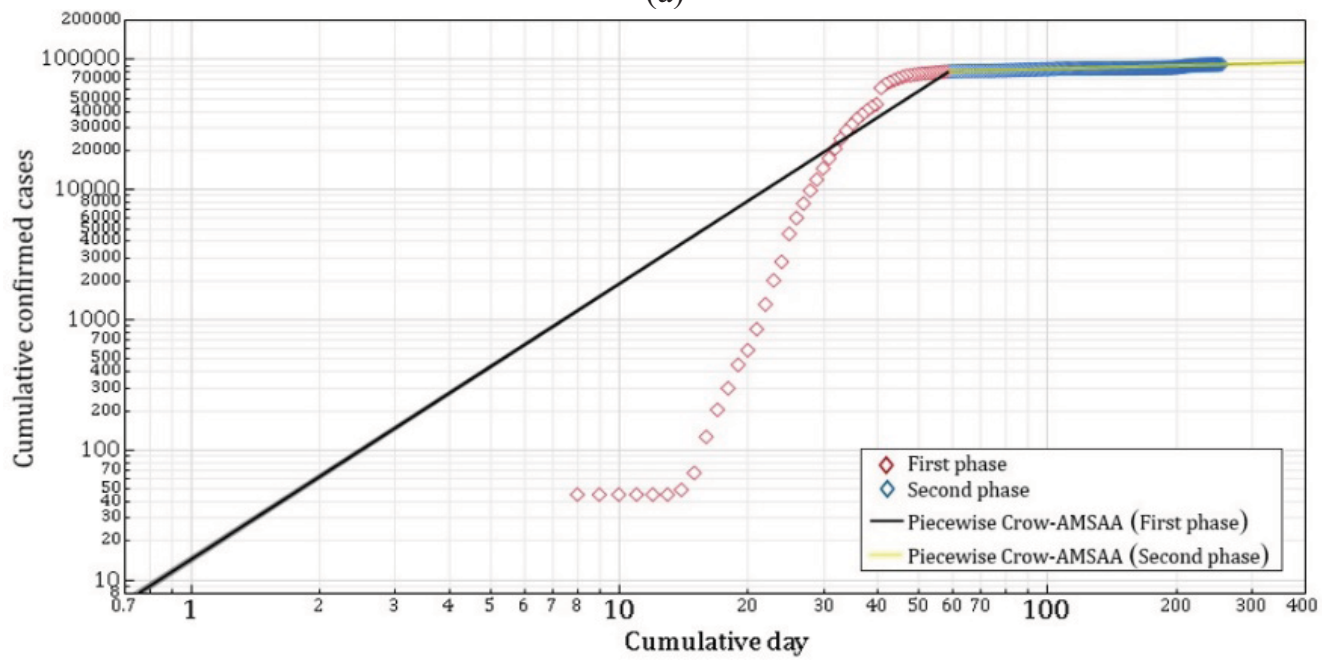

(b)

Figure 3. Cumulative confirmed infected of China; (a) Crow-AMSAA model, (b) Piecewise Crow-AMSAA mode 
other two phases. This means that from the beginning of the outbreak, the speed of the transmission of the disease has been very high in phase one, but in phase two this speed of the transmission decreases. However, in phase three, the speed of transmission increases while its value is less than the value of phase one. Based on the correction actions, it can be concluded that at the beginning of the outbreak, due to its unknown and the factors affecting its speed of transmission, the speed of infectious rate has been very high.

However, by obtaining information about this disease, as well as imposing restrictions and improving health facilities in medical and social centers, the speed of the transmission rate has been decreased. However, in phase three, with the removal of social restrictions imposed by the government, the speed of infectious rate has been increased compared to the previous phases.

But, according to daily reported data from China, the value of the growth parameter $\beta$ in phase one is equal to 2.11 and in phase two is 0.08 . Therefore, due to the fact that in phase one the value of the growth parameter is greater than 1, the speed of the transmission is increasing, while in phase two, because the value of the growth parameter is less than 1, the transmission speed of the disease is decreasing. This shows that the restrictions and requirements imposed by the Chinese government to slow down the transmission speed of the disease have been very effective.

Based on the death reported data from Iran and according to Table 2, the value of the growth parameter $\beta$ in each of the phases one, two, and three is equal to $2.77,1.29$ and 1.39 , respectively, which shows that the daily death rate is increasing in all stages. But in phase two compared to phase one, the death rate decreases and then in phase three death rate increases. Thus, based on the data of the infected people, the correction action by the government and health centers have been somewhat effective but have not been able to reduce the death rate. But according to Chinese data, the value of the growth parameter $\beta$ in phase one is 2.77 , which is greater than 1 , and in phase two, the value of the growth parameter $\beta$ is equal to 0.31 , whichisless than 1 .Thus, the rate of

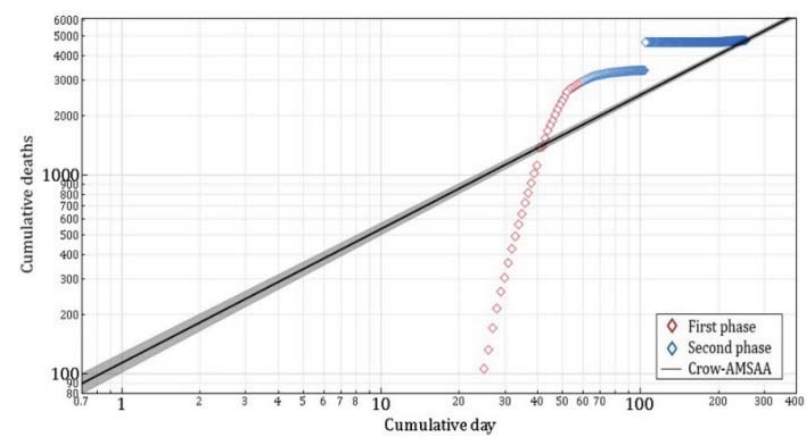

(a)

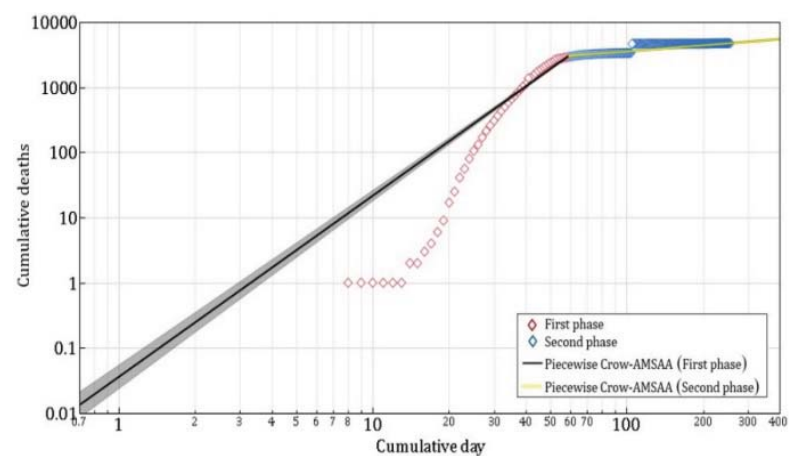

(b)

Figure 4. Cumulative death of China; (a) Crow-AMSAA model, (b) Piecewise Crow-AMSAA modeldeath is increasing

in phase one and decreasing in phase two, indicating that measures taken by Chinese government have been effective.

It is worth mentioning that the growth parameter $\beta$ in the Crow-AMSAA (NHPP) model based on the infected cases data from Iran is 1.42 which indicates the growth of transmission speed of the disease and the growth parameter $\beta$ based on the death data from Iran is 1.47 which indicates the growth of death rate.

However, according to the daily reported data from China, the growth parameter $\beta$ of the confirmed infected case and death is 0.56 and 0.67 , respectively, which in both cases is less than 1 indicating low down the Coronavirus spreading and death rate in China.

In the following, comparison between the real infected cases and the Crow-AMSAA (NHPP) and Piecewise Crow-AMSAA (NHPP) predicted for Iran is shown in Figure 5. Also, the comparison between the real infected cases and the Crow-AMSAA (NHPP) and Piecewise Crow-AMSAA (NHPP) predicted for Coronavirus in China is plotted in Figure 6.Comparison between real infected cases and Piecewise CrowAMSAA (NHPP) prediction shows that, Piecewise Crow-AMSAA (NHPP) model is useful for predicting infected people or deaths due to Coronavirus as well as other epidemics similar to Coronavirus. The limitation of this piecewise Crow-AMSAA (NHPP) method is that there is a need for a manual separation of the data to be applied to find out each different infection phase at each different time period. The good fit of the data is depending on good data separation. So, based on the observation of Iran's cumulative confirmed case and death data 3 phases were considered. But data of China has been divided into 2 phases.

It is worth mentioning that the values and results obtained are only for the data reported by the Iran and published in the website of the World Health Organization (WHO) and taking into account the data from $2 / 19 / 2020$ to $9 / 10 / 2020$. Surely, results will be 
different forthe other data along with the parameters

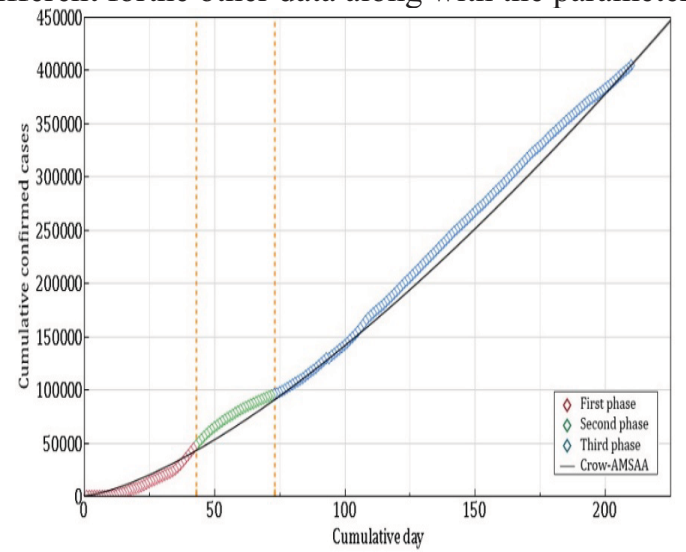

(a)

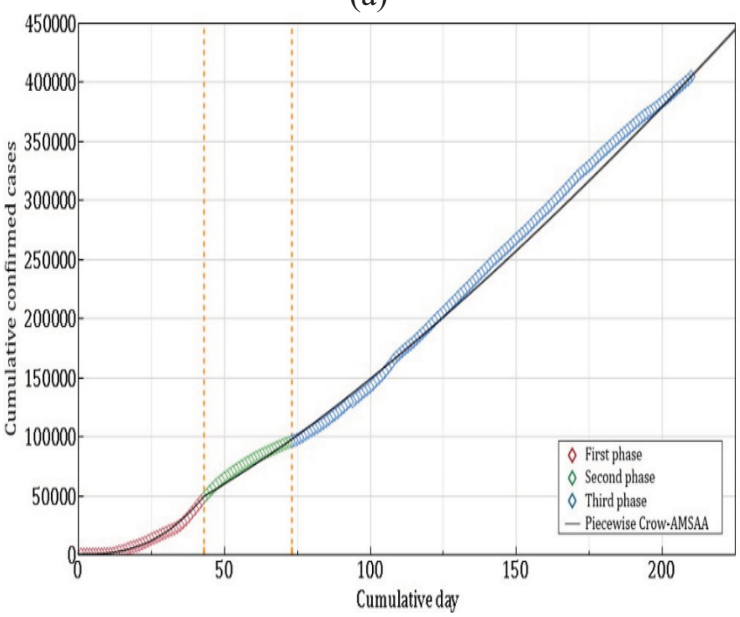

(b)

Figure 5. Iran real infected case versus (a) Crow-AMSAA predicted, (b) Piecewise Crow-AMSAA predictedthat affect it.In addition to the Crow-AMSAA (NHPP) models, other reliability growth models have been developed.Therefore, the study of the the speed of the transmission and death rates by considering other effective parameters in this disease such as demographic conditions, region, genetics, age, climatic conditions, respiratory disease, etc. using Crow- models

AMSAA (NHPP) as well as other reliability growth models are recommended.It is also possible to examine government policy from various social, political and etc. aspects against this disease and its effectiveness using the growth parameter $\beta$ in Crow-AMSAA (NHPP) models.

In this study, Piecewise Crow-AMSAA (NHPP) reliability growth model is used for Coronavirus spreading in Iran. To this purpose, the unknownparameter $\beta$ of the Piecewise CrowAMSAA(NHPP) model has been introduced as the speed of the transmission or death rate for the spread of Coronavirus disease. Then, to evaluate the Piecewise Crow-AMSAA(NHPP) model with the given Coronavirus infection and death reported data, the maximum likelihood estimation technique was used to estimate the effective parameter in reliability growth. The Coronavirus infection and death data from $2 / 19 / 2020$ to $9 / 10 / 2020$ were used in the model for Iran. When the cumulative confirmed new cases and death are plotted and simulation results are compared with experimental data obtained from Coronavirus tests, the model yielded a good estimation. So, the Crow-AMSAA (NHPP) model appears to be useful to predict the infected cases and deaths for a pandemic like Coronavirus. Finally, results obtained from the data of Iran are compared with data of China, as the country of origin of Coronavirus outbreak. In addition to this, the growth of the spread and speed of the transmission or

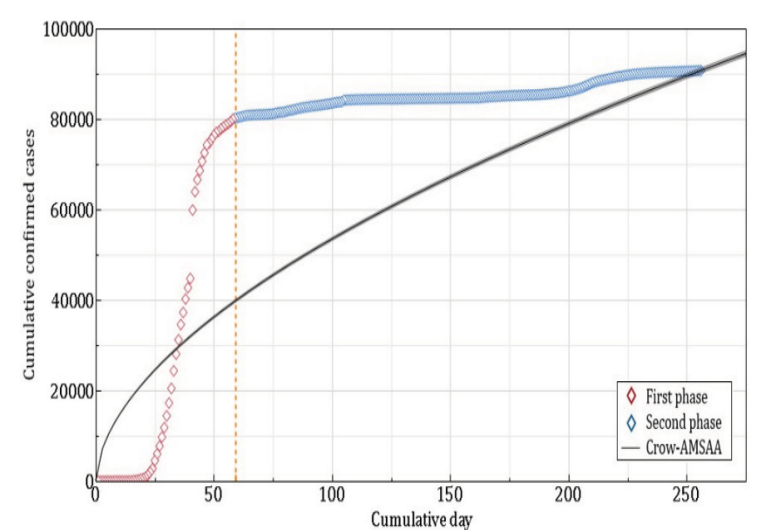

(a)

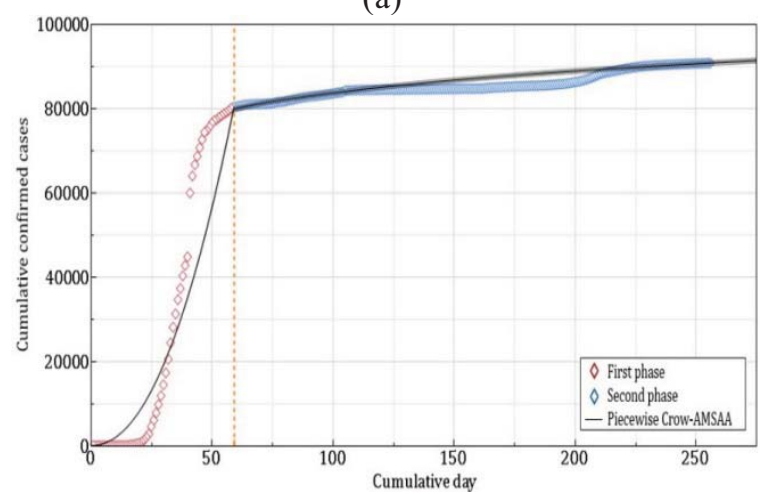

(b)

Figure 6. China real infected case versus (a) Crow-AMSAA predicted, (b) Piecewise Crow-AMSAA predicteddeathrate of these two countries have been studied in relation to each other.

\section{References}

[1] Car, Z., Baressi Šegota, S., Anđelić, N., Lorencin, I. and Mrzljak, V., "Modeling the spread of covid-19 infection using a multilayer perceptron," Computational and Mathematical Methods in Medicine, Vol. 2020, 2020.

[2] Li, L., Yang, Z., Dang, Z., Meng, C., Huang, J., Meng, H., Wang, D., Chen, G., Zhang, J. and Peng, H., "Propagation analysis and prediction of the covid-19," Infectious Disease Modelling, Vol. 5, pp. 282-292, 2020.

[3] Catala, M., Alonso, S., Lacalle, E.A., Lopez, D., Cardona, P.-J. and Prats, C., "Empiric model for short-time prediction of covid-19 spreading," medRxiv, 2020. 
[4] Zareie, B., Roshani, A., Mansournia, M.A., Rasouli, M.A. and Moradi, G., "A model for covid-19 prediction in iran based on china parameters," medRxiv, 2020.

[5] Duane, J., "Learning curve approach to reliability monitoring," IEEE transactions on Aerospace, Vol. 2, pp. 563-566, 1964.

[6] Crow, L.H., "Reliability analysis for complex, repairable systems," Army Material Systems Analysis Activityaberdeen Poving Ground MD, 1975.

[7] Crow, L.H., "Confidence interval procedures for reliability growth analysis,"Army Material Systems Analysis Activityaberdeen Poving Ground MD, 1977.

[8] Crow, L.H., "Confidence interval procedures for the weibull process with applications to reliability growth," Technometrics, Vol. 24, pp. 67-72, 1982.

[9] RANI and Misra, R., "Ml estimates for crow/amsaa reliability growth model for grouped and mixed types of software failure data," International Journal of Reliability, Quality and Safety Engineering, Vol. 11, pp. 329-337, 2004.

[10] Sun, A., Kee, E., Yu, W., Popova, E., Grantom, R. and Richards, D., "Application of crow-amsaa analysis to nuclear power plant equipment performance", in 13th International Conference on Nuclear Engineering, pp. 1-6, 2005.

[11] Barringer, H.P., "Use crow-amsaa reliability growth plots to forecast future system failures," 2006.

[12] Tang, Z., Zhou, W., Zhao, J., Wang, D., Zhang, L., Liu, H., Yang, Y. and Zhou, C., "Comparison of the weibull and the crow-amsaa model in prediction of early cable joint failures," IEEE Transactions on Power Delivery, Vol. 30, pp. 2410-2418, 2015.
[13] Nadjafi, M. and Gholami, P., "Bayesian inference of reliability growth-oriented weibull distribution for multiple mechanical stages systems," International Journal of Reliability, Risk and Safety: Theory and Application, 2021.

[14] Wang, W., Xu, Y. and Hou, L., "Optimal allocation of test times for reliability growth testing with intervalvalued model parameters," Proceedings of the Institution of Mechanical Engineers, Part O: Journal of Risk and Reliability, Vol. 233, pp. 791-802, 2019.

[15] Lee, Y., Ryu, J., Son, K., Song, S., Kim, S. and Park, W., "A study on the reliability growth of multiple launch rocket system using accelerated life testing," Journal of the Korea Institute of Military Science and Technology, Vol. 22, pp. 241-248, 2019.

[16] Nadjafi, M. and Gholami, P., "Developing of reliability growth model based on nonhomogeneous poisson process with normal distribution," Journal of Mechanical Engineering, Vol. 51, pp. 239-248, 2021.

[17] Ranjan, R., Predictions for covid-19 outbreak in india using epidemiological models. Medrxiv. 2020.

[18] Canabarro, A., Tenorio, E., Martins, R., Martins, L., Brito, S. and Chaves, R., "Data-driven study of the covid19 pandemic via age-structured modelling and prediction of the health system failure in brazil amid diverse intervention strategies," medRxiv, 2020.

[19] Liu, Z., Magal, P. and Webb, G., "Predicting the number of reported and unreported cases for the covid-19 epidemics in china, south korea, italy, france, germany and united kingdom," Journal of Theoretical Biology, Vol. 509, p. 110501, 2020. 